\title{
Il Rene Policistico e l'A.I.R.P. onlus
}

\section{Approccio alla terapia idropinica del Rene Policistico}

\author{
N.E. Montemurro
}

S.C. di Nefrologia e Dialisi, Ospedale "SS. Annunziata”, Taranto

Specialista Idrologia Medica

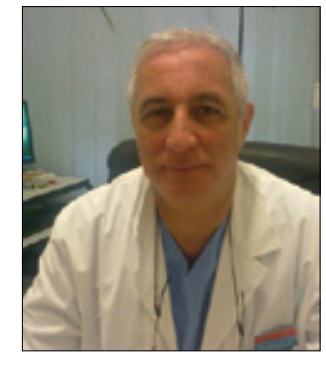

Notoriamente il paziente affetto da Rene Policistico, nel corso della malattia, va incontro a varie complicanze che possono accelerare la comparsa o la progressione verso l'insufficienza renale cronica (IRC). Insieme agli specifici presidi farmacologici e alle norme dietetiche, che insieme restano i cardini della terapia e della prevenzione dell'IRC, è possibile instaurare un approccio con la terapia idropinica (TI) nella cura delle due complicanze che maggiormente beneficiano di un adeguato introito liquido quotidiano: le ripetute infezioni del tratto urinario (UTI) e la nefrolitiasi e/o nefrocalcinosi. Malgrado ci sia un accordo generale di massima circa la quantità d'acqua da somministrare (giusto: somministrare, come un medicinale!) che si aggira intorno ai 2 litri al giorno (1) non v'è pari attenzione da parte dei Nefrologi sul discorso di tipo qualitativo. Studi di Di Paolo et al risalenti al 2000 (2) hanno dimostrato che solo talune acque oligominerali naturali hanno proprietà depurative piuttosto che stimolanti un incremento della diuresi sul rene ammalato: si tratta nella grande maggioranza dei casi di acque naturali ipotoniche con basso tenore in sodio e calcio e quindi a basso residuo fisso (RF). Tenuto conto che per convenzione il RF (ovvero le ceneri di minerali presenti in un litro d'acqua evaporata ed essiccata a $180^{\circ}$ ) deve essere inferiore ai $500 \mathrm{mg} / \mathrm{L}$ per poter definire un'acqua come oligominerale, è comunque bene rifarsi alla definizione di Marotta e Sica (3) che individuavano prima del 1983, molto più correttamente (e meno commercialmente) in $200 \mathrm{mg} / \mathrm{L}$ il RF massimo, che definiva appunto "oligominerale" un'acqua. Nella fattispecie acque con $\mathrm{RF}<200 \mathrm{mg} / \mathrm{L}$ si rivelano molto più efficaci nel favorire un incremento della diuresi anche nel caso del Rene Policistico. In questa particolare situazione patologica, questa categoria di acque si comporta come nel caso della nefrolitiasi (condizione molto meglio studiata e validata da una corposa letteratura scientifica) incrementando il volume urinario. Un'altra complicanza della malattia cistica renale è quella delle frequenti UTI che contribuiscono non poco all'evoluzione verso la franca IR sia direttamente sia indirettamente per il ricorso ad antibiotici la cui nefrotossicità è data non tanto dall'uso del tipo quanto dalla durata del loro impiego. Una TI corretta nella quantità (per sfruttare l'effetto "massa" come avviene nella terapia della calcolosi urinaria) e nella qualità, grazie all'impiego di acque naturali a minima mineralizzazione può arrecare un potenziale beneficio nella cura delle UTI. Questa categoria di acque con un RF intorno ai $20 \mathrm{mg} / \mathrm{L}$ oltre a favorire una detersione delle vie urinarie richiamando sali, di cui sono "avide", corpi batterici e residui litogeni agiscono anche ripristinando una valida peristalsi e riducono situazioni di spasmo e relativo ristagno urinario, fenomeno favorente il perpetuarsi dell'infezione con recidiva o ricaduta della stessa. La caratteristica riduzione della dolenzìa addominale $\mathrm{e}$ delle regioni lombari che affligge sovente i portatori di rene policistici potrebbe essere assimilata a quella dei soggetti calcolotici, che sono stati oggetto di studi con TI con acque ipotoniche, da parte di Urologi francesi in alcune stazioni termali d'Oltralpe negli anni Novanta. Lo scopo raggiungibile con la TI è perlomeno duplice: una detersione continua ostacola fisicamente l'attecchimento degli agenti patogeni alle mucose e diluendo la carica batterica la rende più suscettibile alla terapia antibiotica mirata. Si riducono così il tempo e la dose dell'antibioticoterapia, che resta un indubbio vantaggio 
quando è presente IR, e si consente un ripristino delle naturali difese organiche (Ig secretorie ecc.). Jeanjean e Benoit hanno dimostrato nel 1993 una significativa riduzione $(p<0,05)$ delle reinfezioni in corso di cistite trattando una popolazione con due cicli termali di TI in sei mesi contro una popolazione che aveva effettuato nello stesso periodo ripetuti cicli di antibioticoterapia. In presenza di UTI è corretto impiegare solo acque minerali contenenti meno di $10 \mathrm{mg} / \mathrm{L}$ di nitrati. La modalità di somministrazione dell'acqua oligominerale naturale deve seguire regole il più possibile di tipo "termale". Va senz'altro evitato il carico idrico da $500 \mathrm{~mL}$ in mezz'ora per il rischio di scatenamento di coliche renali o emorragie intracistiche da iposmosi; è anche controindicata l'assunzione di acqua troppo fredda. È corretto educare il paziente a un introito quotidiano con la seguente posologia: $250 \mathrm{~mL}$ da assumere al mattino, a digiuno, in ortostasi o camminando e proseguendo con $250 \mathrm{~mL}$ un'ora prima dei pasti e due ore dopo i pasti; $300 \mathrm{~mL}$ la sera prima di coricarsi e $250 \mathrm{~mL}$ dopo ogni eventuale minzione notturna. Tale trattamento è idoneo anche in caso di Insufficienza Renale Cronica fino al $3^{\circ}$ stadio K/ DOQI (escludendo ovviamente i pazienti con ritenzione idrosalina o franchi edemi). Resta infine da sottolineare che la maggior parte delle acque oligominerali naturali del commercio hanno un tenore in sodio pari o inferiore ai $20 \mathrm{mg} / \mathrm{L}$ che le rende particolarmente vantaggiose quando utilizzate in pazienti con ipertensione arteriosa, da cui molto spesso sono affetti i portatori di Rene Policistico. In conclusione, oltre che essere suggestivo da un punto di vista razionale speculativo può essere utile incoraggiare studi sul trattamento con TI su ampie casistiche di tale tipo di pazienti, date le poche controindicazioni e i possibili benefici che essi ne possono trarre.

\section{Riassunto}

La terapia idropinica è stata da tempo oggetto di interesse nefro-urologico nel trattamento della calcolosi urinaria, mentre poche sono le evidenze scientifiche di un suo razionale nella cura e prevenzione delle complicanze microlitiasiche e delle infezioni urinarie nei pazienti portatori di Rene Policistico. Si propone un approccio alla cura e prevenzione di tali condizioni patologiche con la terapia idropinica, condotta in maniera corretta dal punto di vista dell'Idrologia medica, quale supporto agli altri presidi farmacologici e dietetici.

Parole chiave: Insufficienza renale cronica, Terapia idropinica, Infezioni vie urinarie, Residuo fisso.

\section{Indirizzo degli Autori:}

Nicola Ettore Montemurro, MD

via Di Palma,118

74123 Taranto

nicola.montemurro@libero.it

\section{Bibliografia}

1. Micali F, Porena M, Vespasiani G. La calcolosi urinaria. Wellcome Italia, 1979; 105.

2. Agostini G. Manuale di Medicina Termale. II Edizione. 2000 Torino: Archimedica, 2000; 119.

3. Marotta D, Sica C. Classificazione delle acque minerali italiane. Giorn. di Chim. ind. ed applicata, 1929; XI (6): 276. 